\title{
AN OBSERVATIONAL METHOD FOR THE LOWER LATITUDE MERIDIAN CIRCLE (LLMC) WITH A CCD CAMERA
}

\author{
Mao Wei, Fan Yu, Peng Qinyu, \\ Li Zhiming, Ji Kaifan, and $\mathrm{Hu}$ Xiaochun \\ Yunnan Observatory \\ Chinese Academy of Sciences \\ P.0.Box 110 \\ Kunming, Yunnan Province \\ China
}

ABSTRACT. A principle for absolute determination of an object' position by means of LLMC with a CCD camera and the method for finding the error from the instrument and the CCD chip are described in this paper. The observational power and application of the LLMC with a CCD are also discussed.

\section{INTRODUCTION}

CCD has been widely used in the field of astronomy for its high quantum efficiency, relative measurement accuracy and observational efficiency. Lately, in astrometry, some meridian circles[1] with a CCD camera have already been used as the connection of the Hipparcos frame and the radio source frame. This will play an important role in the construction of a quasiinertial celestial reference frame.

In Yunnan Observatory, after a CCD is attached to the LLMC which will be put into operation in the near future for the large mount of position work the observation will combine closely the absolute observation and relative one, as well as the large angle and small one in astrometry. So, the LLMC-CCD system will be used to construct directly a quasi-inertial reference frame through the ground-based observation.

\section{THE LLMC-CCD SYSTEM}

2.1. The Observational Principle and Instrumental Errors of the LLMC-CCD System

The LLMC is a $\mathrm{R}-\mathrm{C}$ reflector with diameter $\mathrm{d}=400 \mathrm{~mm}$ and focal length $F=4.8 \mathrm{~m}$. According to its observational principle[2], the instrument will observe the same star alternately in the prime vertical and in meridian so that the instantaneous latitude and absolute azimuth can be obtained

Because the observation set is made with rotated torizonal axis of the instrument, then some instrumental errors ( sich as the zenith error of the graduated circle and collimation error) 
can be eliminated. On the other hand, level error, irregular error of the pivot, flexure of the tube ( including the displacements and rotations of the prime reflector and the by-reflector as well as the micrometer) and the instrumental orientating error all can be determined by a series of our new explored methods. The accuracy of the determination for all of errors will be better than $+0 " .01$.

It is the most possible that the CCD chip which will be attached to the LLMC has $1024 \times 1024$ pixels in frame format, with the pixel size of 15 microns square, the field of view for the chip is 11' $x 11^{\prime}$ relatively. The instrument will be capable of measuring a star or an extragalactic object down to magnitude of $\mathrm{V}=17 \mathrm{mag}$ and the relative positional accuracy to any pairs of images will be \pm 0.02 pixel in the field of view.

\subsection{The Errors of the CCD Chip}

The errors due to the manufacturing of the CCD chip include the nonperpendicular and inequality of the scales between the $x$-axis and $y$-axis. The nonperpendicular between the CCD plane and the light axis (one of the installation errors) is also one source of the error. The other installation error is the unparallism between the $y$-axis and the projection of the meridian. All these errors can be solved by observing the same region in meridian and prime vertical[3].

\section{THE OBSERVATION MODE OF THE LLMC-CCD SYSTEM}

In the course of the observation in meridian with CCD chip tracking a celestial object at the rate of $15^{\prime \prime} \cos / \mathrm{sec}$ during the exposure of 30 seconds, the time and the zenith distance of the object passing through meridian will be

$$
\begin{aligned}
& \mathrm{Tm}=\left(\bar{t}_{i}+\bar{t}_{i}^{\prime}\right) / 2+\left(\bar{b}_{i}-\bar{b}_{i}\right) R_{b} \sec \delta_{0} / 30+\left(x^{\prime}-x\right) R_{x} \sec \delta_{\bullet} / 30, \\
& \mathrm{Zm}=\left(M-M^{\prime}\right) / 2+\left(y^{\prime}-y\right) R_{y} / 2+\Delta Z_{c},
\end{aligned}
$$

where, $(x, y)$ and $\left(x^{\prime}, y^{\prime}\right)$ are the measured coordinates of the object before and after the rotation of horizontal axis respectively; $b_{i}$ and $b_{i}$ measured by a position sensor are the positions of the CCD chip at the moments $t_{i}$ and $t_{i}^{\prime} ; M$ and $M^{\prime}$ are the readings of the graduated circle; $R_{b}, R_{x}$ and $R_{y}$ are the scales, $\Delta Z_{c}$ is the curvature correction for the observed object.

In the course of the observation in prime vertical with CCD chip tracking the object in the horizontal direction and with the telescope tracking in the vertical direction during the exposure for the same duration of that as in meridian, the time and the zenith distance through prime vertical can be expressed as

$$
\begin{aligned}
T_{\text {ow }}= & \left(\bar{t}_{i}+\bar{t}_{i}^{\prime}\right) / 2 \div\left[\left(\bar{b}_{i}^{\prime}-\bar{b}_{c}\right) R_{b} \div\left(x^{\prime}-x\right) R_{x}\right] /\left(\sin \varphi_{0} \sin Z_{e w}^{\prime}\right) / 30+\Delta t_{c}, \\
Z_{e w}= & \left(\bar{M}_{i}-\bar{M}_{i}^{\prime}\right) / 2+\left(y^{\prime}-y\right) R_{y} / 2+\left[\left(\bar{b}_{i}^{\prime}-\bar{b}_{i}\right) R_{b}+\left(x^{\prime}-x\right) R_{x}\right] \cos \varphi_{0} /\left(\sin \varphi_{0}\right. \\
& \left.\sin Z_{e w}^{\prime}\right) / 2+\Delta Z_{c}(e w),
\end{aligned}
$$


where, $M_{i}$ and $M_{i}^{\prime}$ are the readings of the graduated circle at the recorded times $t_{i}$ and $t_{i}$ respectively; $Z_{\text {ew }}$ of above two equations is the approximate value of the zenith distance of the object in prime vertical; $\Delta t_{c}$ and $\Delta Z_{c(e w)}$ are curvature corrections for the object during the passing time. The others have the same meanings as those explained for the meridian case.

\section{THE OBSERVATIONAL POWER AND APPLICATION OF THE LLMC-CCD SYSTEM}

\subsection{Direct Construction of A Quasi-inertial Reference Frame}

The absolute positions of extragalactic objects and the absolute proper motions of the stars relative to an extragalactic object can determined through the observation using the method mentioned above: After reasonable observation of extragalactic objects and stars at 3 epochs in period of nearly 10 years, the corrections to precessional constant ( including planetary precessional constant ) and nutational constant can be determined absolutely, thus, a quasi-inertial reference frame can be constructed directly.

\subsection{Observation of Asteroids}

About 3,000 asteroids can be observed in their whole orbit by use of the LLMC-CCD system, so that a dynamics frame be constructed. At the same time, some useful data will also be provided for dynamics of the solar system.

\subsection{Observation of Some Special Celestial Objects}

The instrument can be employed to obtain absolute positions, absolute proper motions, luminosities and annual parallaxes of some star cluster, double stars and variables. This will make contribution to the research of Stellar Astronomy and the Galaxy

\subsection{To Obtain a Practical Atmospheric Refraction Table}

By use of the observations in prime vertical and meridian, the instantaneous atmospheric refraction and the slope of air isopycnic layer can be determined absolutely. If the observed data of astrolabe are compared with that of the LLMC-CCD system, the difference of atmospheric refraction constants in the directions of south and north of the zenith as well as that in prime vertical and meridian directions can be obtained. So, it is pledged to research into the rule of atmospheric changes and to construct a practically measured atmospheric refraction table corresponding to an observing station.

\subsection{To Provide Some Useful Data for Geophysics}

Through the reduction of the observed data at different time, the variety of the plumb line can be studied and some valuable information for geophysics can also be provided. 
64

\section{REFERENCE:}

[1] Stone,R.C., 1990, Inertial Coordinate System On The Sky, Symposium No. 141, P369.

[2] Mao Wei, et al.,1987, Method of Meridian Astronony, Science Press (in Chinese).

[3] Mao Wei, et al., 1991, Publications of Yunnan Observatory, No.1, 61 . 